\title{
Rodízio de Opioides: uma Análise Descritiva
}

https://doi.org/10.32635/2176-9745.RBC.2021v67n2.1179

\author{
Opioid Switching: a Descriptive Analysis \\ Rotación de Opioides: un Análisis Descriptivo
}

Simone Garruth dos Santos Machado Sampaio'; Luciana Branco da Motta²; Célia Pereira Caldas ${ }^{3}$

RESUMO

Introduçáo: $\mathrm{O}$ uso de opioides deve ser individualizado e a troca por outro opioide pode ser necessária (rodízio de opioide). Objetivo: Identificar como foi realizado o rodízio de opioide e se o efeito desejado foi atingido em pacientes internados em uma unidade especializada em cuidados paliativos oncológicos. Método: Análise post hoc do estudo de perfil de pacientes internados em um hospital público de cuidados paliativos oncológicos no Rio de Janeiro, entre setembro e novembro de 2016. As internaçóes foram acompanhadas longitudinalmente por revisão de prontuário com coleta diária da escala verbal numérica $(\mathrm{EVN})$. A dor foi considerada controlada quando EVN =0. Doses, via de administração e rodízio (fármaco e motivo) dos opioides foram observados. O tempo para controle da dor foi calculado quando este foi o motivo. Resultados: Foram observados 104 rodízios de opioides em 90 internações (22,5\%), sendo 49\% entre opioides fortes e $43 \%$ de fraco para forte. Principais motivos foram dor (40\%) e dispneia (36\%). O tempo para EVN = 0 foi 1,6 dias (+/-1,8; IC95\% 1,0-2,1), sendo mais demorado na troca por metadona (média 2,7 dias +/-2,5; IC95\% 1,0-4,4). Comparando a dose de morfina oral por equipotência analgésica, houve aumento de $10 \%$ na dose do opioide de destino, sendo esse aumento maior quando no rodízio por dispneia (38\%). Conclusáo: Embora o controle de dor tenha sido superior ao descrito por outros trabalhos, o aumento da dose equipotente do opioide não é corroborado por protocolos. Maior vigilância e outros estudos são recomendados na unidade.

Palavras-chave: Cuidados Paliativos; Manejo da Dor; Analgésicos Opioides/administração \& dosagem; Conduta do Tratamento Medicamentoso.

\section{ABSTRACT}

Introduction: The use of opioids must be individualized and changing for another opioid may be necessary (opioid switching). Objective: Identify how the opioid switching was performed and whether the desired effect was achieved in patients admitted at a public palliative oncologic care specialized hospital. Method: Post hoc analysis of the profile study of patients admitted to a public oncologic palliative care hospital in Rio de Janeiro between September and November 2016. Hospitalizations were followed longitudinally by reviewing the charts with daily collection of the numeric rating scale (NRS). Pain was considered controlled when NRS = 0 . Doses, route of administration, switch (drugs and motif) of the opioids were observed. The time for pain control was calculated when this was the reason. Results: 104 opioid switching were observed in 90 hospitalizations (22.5\%), 49\% of which were strong opioids and $43 \%$, from mild to strong. Main reasons were pain $(40 \%)$ and dyspnea $(36 \%)$. The time to NRS $=0$ was 1.6 days $(+/-1.8 ; 95 \%$ CI $1.0-2.1)$, taking longer to switch to methadone (mean 2.7 days $+/-2.5$; $95 \%$ CI $1.0-4.4$ ). Comparing the dose of oral morphine by analgesic equipotency, a $10 \%$ increase in the target opioid dose occurred, and when rotating due to dyspnea (38\%), the increase was greater. Conclusion: Although pain control was higher than described in other studies, the increase in the equipotent dose of opioid is not corroborated by protocols. Extensive surveillance and other studies are recommended in the unit.

Key words: Palliative Care; Pain Management; Analgesics, Opioid/ administration \& dosage; Medication Therapy Management.
RESUMEN

Introducción: El uso de opioides debe ser individualizado y puede ser necesario cambiarlo por otro opioide (rotación de opioides). Objetivo: Identificar cómo se realizó la rotación de opioides y si el efecto deseado se logró en pacientes ingresados en una unidad especializada en cuidados oncológicos paliativos. Método: Análisis post hoc del estudio de perfil de pacientes ingresados en un hospital público de cuidados paliativos de oncología en Río de Janeiro, entre septiembre y noviembre de 2016. Las hospitalizaciones fueron seguidas longitudinalmente mediante la revisión de los registros médicos con la recopilación diaria de la Escala Numérica Verbal (ENV). El dolor se consideró controlado cuando $\mathrm{ENV}=0$. Se observaron dosis, vía de administración, rotación (fármacos y motivo) de los opioides. El tiempo para el control del dolor se calculó cuando esta fue la razón. Resultados: Se observaron 104 ruedas de opioides en 90 hospitalizaciones $(22,5 \%)$, con $49 \%$ entre opioides fuertes y $43 \%$ de débiles a fuertes. Las razones principales fueron dolor $(40 \%)$ y disnea $(36 \%)$. El tiempo para $\mathrm{ENV}=0$ fue de 1,6 días (+/-1,8; IC del 95\%: 1,0-2,1), y tomó más tiempo cambiar a metadona (promedio 2,7 días +/-2,5; IC 95\% 1,0-4,4). Comparando la dosis de morfina oral para la equipotencia analgésica, hubo un aumento del $10 \%$ en la dosis de opioides objetivos, este aumento fue mayor al rotar debido a la disnea (38\%). Conclusión: Aunque el control del dolor fue superior al descrito por otros estudios, el aumento en la dosis equipotente de opioide no es compatible con los protocolos. Se recomienda mayor vigilancia y otros estudios en la unidad.

Palabras clave: Cuidados Paliativos; Manejo del Dolor; Analgésicos Opioides/administración \& dosificación; Administración del Tratamiento Farmacológico.

'Instituto Nacional de Câncer José Alencar Gomes da Silva (INCA). Unidade de Cuidados Paliativos do Hospital do Câncer IV (HCIV). Rio de Janeiro (RJ), Brasil. E-mail: simonegarruth@gmail.com. Orcid iD: https://orcid.org/0000-0001-5537-7399

2,3Universidade do Estado do Rio de Janeiro (UERJ). Núcleo de Atenção ao Idoso (NAI). Rio de Janeiro (RJ), Brasil. E-mails: lumotta@uerj.br; celpcaldas@hotmail.com. Orcid iD: https://orcid.org/0000-0001-9959-9719; Orcid iD: https://orcid.org/0000-0001-6903-1778

Endereço para correspondência: Simone Garruth dos Santos Machado Sampaio. INCA/Unidade de Cuidados Paliativos do HC IV. Rua Visconde de Santa Isabel, 274 - Vila Isabel. Rio de Janeiro (RJ), Brasil. CEP 20560-120. E-mail: simonegarruth@gmail.com 


\section{INTRODUÇÃO}

O uso de analgésicos opioides é uma das bases para o controle da dor e da dispneia em pacientes em cuidados paliativos $^{1}$. Para orientar seu uso no tratamento da dor, a Organização Mundial da Saúde (OMS) ${ }^{2}$ criou a "escada analgésica”, classificando os opioides em fracos ou fortes segundo sua potência. O objetivo desse protocolo é auxiliar o profissional a avaliar e adaptar as medicaçôes utilizadas conforme a necessidade do paciente em cada momento, de forma sequencial e progressiva ${ }^{2}$.

A terapia com opioides precisa ser individualizada, respeitando-se a dose necessária para cada paciente em cada momento. Em algumas situaçóes, a troca por outro opioide deve ser considerada, o que se denomina "rodízio de opioide". São elas: efeito adverso intolerável durante a titulação, controle analgésico ruim apesar do ajuste da dose, interaçóes fármaco-fármaco limitantes, necessidade de troca de via de administração, benefícios clínicos específicos de alguns opioides (morfina - dispneia; oxicodona - dor neuropática) ${ }^{3}$.

O novo medicamento deve ser prescrito em uma dose que não resulte em efeito adverso ou abstinência e tenha eficácia no controle do sintoma. Para auxiliar nesse processo, existem vários instrumentos desenvolvidos para estabelecer equipotência analgésica entre os opioides ${ }^{4,5}$.

Uma avaliação do perfil de pacientes internados, de medicamentos com potencial analgésico utilizados e do controle de dor atingido, foi desenvolvida em uma unidade pública de cuidados paliativos oncológico na cidade do Rio de Janeiro ${ }^{6}$. A partir da observaçáo do grande número de rodízios de opioide no período, uma análise posterior desse grupo foi desenvolvida. O presente estudo tem como objetivo identificar como foi realizado o rodízio de opioide e se atingiu-se o efeito desejado em pacientes internados em uma unidade especializada em cuidados paliativos oncológicos.

\section{MÉTODO}

Entre $1^{\circ}$ de setembro e 30 de novembro de 2016, foi realizado um estudo transversal para avaliaçáo do perfil de pacientes e do seu tratamento medicamentoso em um hospital público de cuidados paliativos oncológicos na cidade do Rio de Janeiro ${ }^{6}$. Uma análise post hoc com foco no rodízio de opioide foi realizada e descrita neste trabalho.

$\mathrm{Na}$ ocasião, todas as internaçóes foram acompanhadas por revisão diária de prontuário. A dor foi quantificada pela Escala Verbal Numérica $(\mathrm{EVN})^{7-10}$, aplicada e registrada em prontuário na admissão e nas evoluçóes médicas de rotina. Os quatro médicos de rotina integravam a equipe clínica de cuidados paliativos oncológicos da unidade por pelo menos seis anos e foram previamente retreinados para uso das escalas.

Os opioides padronizados na unidade na ocasiấo eram tramadol, codeina, morfina, metadona, oxicodona e fentanil transdérmico. Suas doses e a via de administração prescritas foram observadas. Foram considerados todos os rodízios de opioide, independente da potência. A motivaçáo da troca do opioide foi obtida a partir de informaçôes contidas na evolução médica.

Para fins de análise do estudo, as doses dos opioides foram convertidas para morfina oral segundo a equipotência analgésica, seguindo as orientaçôes da National Comprehensive Cancer Network ${ }^{4}$.

Para análise do rodízio de opioide, a unidade utilizada foi a internação - chamada de episódio - e não o paciente. Foi realizada a análise descritiva das variáveis coletadas com cálculo de frequências, medidas de tendência central e dispersão. As medidas de associação foram calculadas utilizando os testes qui-quadrado para variáveis categóricas, Mann-Whitney para as variáveis numéricas e Wilcoxon pareado para variáveis numéricas pareadas. Todas as variáveis numéricas coletadas possuem distribuição não normal segundo o teste de Shapiro-Wilk. Foi utilizado o programa estatístico R.

O projeto foi aprovado pelo Comitê de Ética em Pesquisa do Instituto Nacional de Câncer José Alencar Gomes da Silva (INCA) e do Hospital Universitário Pedro Ernesto/Universidade do Estado do Rio de Janeiro (Uerj), sob o número de parecer 1.630.518 (CAAE 54919016.4.0000.5274).

\section{RESULTADOS}

Entre $1^{\circ}$ de setembro e 30 de novembro de 2016, 399 pacientes foram internados na unidade, perfazendo um total de 461 episódios de internação. Foram utilizados analgésicos opioides em 400 desses episódios, ocorrendo rodízio em 90 (22,5\%).

A dor foi o principal causa de internação. Considerando todos os episódios, a dor estava presente em $44 \%$ (176) das internaçóes e motivou 19\% (76) de todas as internaçôes. Considerando apenas os episódios em que foram observados rodízio de opioide, a dor foi relatada em 47,8\% (43) das admissóes, e sendo o principal motivo em $26,7 \%$ (24).

O tempo de internação médio foi de oito dias. Analisando separadamente os episódios com e sem a ocorrência de rodízio de opioide, observou-se uma internação mais prolongada quando houve rodízio de opioide (Tabela 1).

Dos 90 episódios em que ocorreu rodízio de opioide, em dez, foi realizado um segundo rodízio e, em outros 
dois, um terceiro, perfazendo um total de 104 rodízios. Dispneia e dor foram os principais sintomas que motivaram o rodízio de opioide, sendo responsáveis por $77 \%$ (80) deles. A Tabela 2 descreve o tipo e a razão do rodízio.

Em todos os casos de rodízio justificados por insuficiência renal, houve substituição de morfina por metadona. Nos dois rodízios motivados por tosse, houve troca de tramadol por codeína. Nos rodízios motivados por dispneia, a troca foi para morfina. Quando a facilidade de via de administração era o objetivo, a troca foi para fentanil transdérmico.
Nos rodízios motivados por dor em uso opioides fortes, as trocas se distribuíram entre os quatro medicamentos padronizados na unidade: em dois, o fentanil foi substituído; em quatro, a metadona; em nove, a morfina; e, em cinco, a oxicodona.

Entre os seis rodízios motivados pela suspeita de efeito adverso, os sintomas apresentados foram delirium, náuseas e sonolência. Para o sintoma náusea, nos dois casos, o medicamento substituído foi tramadol: um para codeína com melhora do sintoma e outro para morfina com manutenção da náusea. Nos dois casos de delirium, o opioide de origem foi a morfina, um com troca para

Tabela 1. Duração (dias) dos episódios de internação com uso de analgésico opioide, entre $1^{\circ}$ de setembro e 30 de novembro de 2016 , em uma unidade de cuidado paliativo oncológico no Rio de Janeiro (RJ)

\begin{tabular}{l|c|c|c|c|c|c|}
\hline & \multicolumn{2}{|c|}{ Episódios totais $(\mathbf{n = 4 0 0 )}$} & $\begin{array}{c}\text { Com rodízio de opioide } \\
(\mathbf{n = 9 0 )}\end{array}$ & \multicolumn{2}{c}{$\begin{array}{c}\text { Sem rodízio de opioide } \\
(\mathbf{n = 3 1 0 )}\end{array}$} \\
\hline & Média (+/-DP) & IC95\% & Média (+/-DP) & IC95\% & Média (+/-DP) & IC95\% \\
\hline $\begin{array}{l}\text { Tempo de } \\
\text { internação } \\
\text { (dias) }\end{array}$ & $8,4(+/-7,3)$ & $7,7-9,2$ & $10,4(+/-7,8)$ & $8,8-12,1$ & $7,9(+/-7,1)$ & $7,1-8,7$ \\
\cline { 2 - 7 } & & & & \multicolumn{2}{|c|}{ p-valor* 0,00} & \\
\hline
\end{tabular}

Legenda: DP = Desvio-padrão; IC = Intervalo de confiança; *Teste Mann-Whitney.

Tabela 2. Descrição dos rodízios de opioides realizados nos episódios de internação, entre $1^{\circ}$ de setembro e 30 de novembro de 2016 , em uma unidade de cuidado paliativo oncológico no Rio de Janeiro (RJ)

\begin{tabular}{|c|c|c|c|c|c|c|c|c|c|}
\hline \multirow[t]{2}{*}{ Motivo do rodízio } & \multicolumn{2}{|c|}{$\begin{array}{l}\text { Opioide fraco } \\
\text { para opioide } \\
\text { forte }\end{array}$} & \multicolumn{2}{|c|}{$\begin{array}{c}\text { Opioide forte } \\
\text { para opioide } \\
\text { forte }\end{array}$} & \multicolumn{2}{|c|}{$\begin{array}{l}\text { Opioide forte } \\
\text { para opioide } \\
\text { fraco }\end{array}$} & \multicolumn{2}{|c|}{$\begin{array}{l}\text { Opioide fraco } \\
\text { para opioide } \\
\text { fraco }\end{array}$} & \multirow{2}{*}{ Total } \\
\hline & $\mathbf{n}$ & $\%$ & $\mathbf{n}$ & $\%$ & $\mathbf{n}$ & $\%$ & $\mathbf{n}$ & $\%$ & \\
\hline \multicolumn{10}{|c|}{ Primeiro rodízio } \\
\hline Dor & 21 & 48,9 & 18 & 43,9 & & & & & 39 \\
\hline Dispneia & 20 & 46,5 & 10 & 24,4 & & & & & 30 \\
\hline Insuficiência renal & & & 10 & 24,4 & & & & & 10 \\
\hline Efeito adverso & 1 & 2,3 & 1 & 2,4 & 3 & 100 & 1 & 33,3 & 6 \\
\hline $\begin{array}{l}\text { Facilidade de via de } \\
\text { administração }\end{array}$ & 1 & 2,3 & 2 & 4,9 & & & & & 3 \\
\hline Tosse & & & & & & & 2 & 66,7 & 2 \\
\hline Total & 43 & & 41 & & 3 & & 3 & & 90 \\
\hline \multicolumn{10}{|c|}{ Segundo rodízio } \\
\hline Dor & & & 2 & 20 & & & & & 2 \\
\hline Dispneia & 2 & 100 & 5 & 50 & & & & & 7 \\
\hline $\begin{array}{l}\text { Facilidade de via de } \\
\text { administração }\end{array}$ & & & 3 & 30 & & & & & 3 \\
\hline Total & 2 & & 10 & & & & & & 12 \\
\hline \multicolumn{10}{|c|}{ Terceiro rodízio } \\
\hline Dispneia & & & 2 & 100 & & & & & 2 \\
\hline Total & & & 2 & & & & & & 2 \\
\hline
\end{tabular}


fentanil transdérmico e outro para tramadol (ambos sem melhora do quadro). Nos dois casos de sonolência, o rodízio de fentanil para tramadol, não gerou mudança no quadro, já no rodízio de morfina para tramadol, houve melhora do sintoma.

Quando o motivo do rodízio foi dor, esta foi controlada em uma média de 1,6 dias (+/-1,8, IC95\% 1,0-2,1), sendo mais rápida entre os rodízios de opioide fraco para forte $(2,1$ dias $+/-2,1$, IC95\% 1,1-3,1) do que entre opioides fortes ( 1 dia $+/$ - 1 , IC95\% 0,5-1,5), porém a diferença não se mostrou estatisticamente significante (p-valor 0,11). A Figura 1 apresenta essas médias.

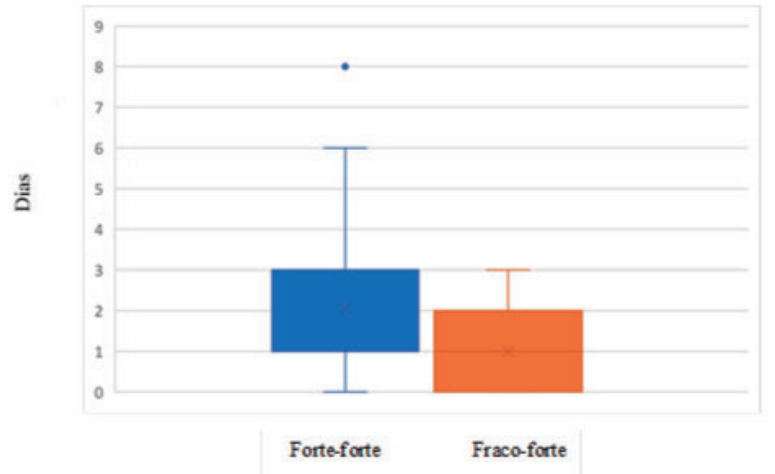

Figura 1. Boxplot* do tempo (em dias) necessário para controle da dor após rodízio de opioide motivado por dor, segundo potência analgésica, nos episódios de internação, entre $1^{\circ}$ de setembro e 30 de novembro de 2016, em uma unidade de cuidado paliativo oncológico no Rio de Janeiro (RJ)

Legenda: ${ }^{*}$ Teste Mann-Whitney, p-valor 0,11.

Em apenas três dos 39 rodízios motivados por dor, o sintoma não obteve controle. Em dois deles, o paciente evoluiu a óbito (ambos com troca de tramadol para morfina) e o outro recebeu alta (troca de fentanil para oxicodona), mantendo relato de dor em todos os dias.
Avaliando isoladamente cada analgésico opioide iniciado como substituto para o controle do sintoma dor, o uso da morfina esteve associado com um controle mais rápido da dor e a metadona com controle mais demorado (Tabela 3).

Considerando a conversão segundo a equipotência analgésica para morfina oral das doses prescritas antes e após o rodízio dos opioides, foi observado um aumento estatisticamente significativo na dose de destino. A diferença nas doses, separadas por motivo do rodízio, foi calculada e está descrita na Tabela 4.

Tabela 3. Tempo para controle da dor (em dias), segundo medicamento, após rodízio de opioide motivado por dor nos episódios de internação, entre $1^{\circ}$ de setembro e 30 de novembro de 2016, em uma unidade de cuidado paliativo oncológico no Rio de Janeiro (RJ)

\begin{tabular}{|c|c|c|c|}
\hline \multirow[t]{2}{*}{ Opioide } & \multirow[t]{2}{*}{$\mathrm{n}$} & \multicolumn{2}{|c|}{$\begin{array}{l}\text { Tempo para controle da } \\
\text { dor após o rodízio (dias) }\end{array}$} \\
\hline & & Média (+/-DP) & IC 95\% \\
\hline Morfina & 20 & $1,05(+/-1,2)$ & $0,5-1,6$ \\
\hline Metadona & 11 & $2,7(+/-2,5)$ & $1,0-4,4$ \\
\hline Oxicodona & 7 & $1,3(+/-0,8)$ & $0,6-2,0$ \\
\hline Fentanil & 1 & 1 & \\
\hline
\end{tabular}

Legendas: $\mathrm{DP}=$ Desvio-padrăo; $\mathrm{IC}$ = Intervalo de confiança.

\section{DISCUSSÃO}

O rodízio de opioide é alvo de grande discussão entre especialistas em dor e cuidados paliativos. Sua necessidade reflete a presença de sintoma com maior complexidade para controle, requerendo maior expertise da equipe profissional que conduz o caso.

Outros autores estudaram casos nos quais foi necessário realizar o rodízio de opioide. Os achados quantitativos descrevem ser minoria dos casos tanto em ambiente

Tabela 4. Dose equivalente de morfina oral dos opioides utilizados nos rodízios dos episódios de internação, entre $1^{\circ}$ de setembro e 30 de novembro de 2016, em uma unidade de cuidado paliativo oncológico no Rio de Janeiro (RJ)

\begin{tabular}{r|c|c|c|c|}
\hline & $\begin{array}{c}\text { Opioide origem - equivalente } \\
\text { morfina (mg) }\end{array}$ & $\begin{array}{c}\text { Opioide destino - equivalente } \\
\text { morfina (mg) }\end{array}$ \\
\cline { 2 - 5 } Global & $\begin{array}{c}\text { Média (+/-DP) } \\
\text { IC 95\% }\end{array}$ & $\begin{array}{c}\text { Média (+/-DP) } \\
\text { IC 95\% }\end{array}$ & p-valor \\
\hline Motivo do rodízio & & & & \\
Dispneia & $87,0(+/-170,0)$ & $97,7-163,9$ & $143,6(+/-168,9)$ & $110,8-176,4$ \\
Dor & $175,3(+/-211,2)$ & $108,6-241,9$ & $189,0(+/-196,3)$ & $137,0-250,9$ \\
Efeito adverso & $70,0(+/-58,8)$ & $8,2-131,7$ & $36,2(+/-24,7)$ & $10,2-62,2$ \\
Insuficiência renal & $104,4(+/-78,6)$ & $48,2-160,6$ & $76,0(+/-33,8)$ & $51,8-100,2$ \\
Tosse & $12,5(+/-7,5)$ & $-82,8-107,8$ & $20,0(+/-9,8)$ & $-68,9-108,9$ \\
Via de administração & $180,8(+/-209,2)$ & $-78,9-440,6$ & $172,2(+/-201,5)$ & $-78,0-422,4$ \\
\hline
\end{tabular}

Legendas: DP = Desvio-padrão; IC = Intervalo de confiança. 
hospitalar quanto domiciliar, em resultados próximos aos encontrados neste estudo. Uma pesquisa conduzida por Corli et al. ${ }^{11}$ avaliou o rodízio entre opioides fortes, que ocorreu em 15,9\% dos pacientes avaliados. Em um estudo com 1.141 pacientes em cuidados paliativos oncológicos domiciliar com uso de opioide forte, a necessidade de rodízio ocorreu em $17 \%$ dos $\operatorname{casos}^{12}$. No presente estudo, o rodízio entre opioides de qualquer potência correspondeu a 22,5\% dos episódios. Ao fazer um recorte considerando apenas o rodízio entre opioides fortes, o evento foi observado em 13,3\% (53) dos episódios.

Alguns autores descrevem a dor como principal motivo para o rodízio de opioide. Isso também foi observado neste estudo, sendo responsável por 39,4\% (41) das trocas como um todo. Ao se restringir apenas a rodízio entre opioides fortes, o valor é de 37,7\% (20 episódios). A frequência observada por outros autores é mais elevada, variando de $52 \%$ a $83 \%^{11,13}$. O controle da dor obtido no presente estudo foi superior ao descrito na literatura. Apenas em três episódios (7\%), o sintoma não foi controlado. Embora estudos mostrem resultados positivos, a taxa de insucesso chega a $49 \%^{11,13,14}$.

Embora proporcionalmente a quantidade de rodízio de opioide encontrada seja semelhante à observada por outros autores, ao analisar exclusivamente os casos em que foram motivados por dor, os resultados proporcionais verificados são divergentes. Nos estudos mencionados anteriormente, foram descritos apenas os medicamentos opioides sem menção ao uso de medicamentos adjuvantes no controle da dor, o que pode justificar achados quantitativos diferentes. Pela análise do perfil da unidade de onde originaram esses dados ${ }^{6}$, o uso de analgésicos comuns e adjuvantes foi maior do que os descritos por outros autores tanto em frequência quanto em dose. Tal diferença pode ser uma das razóes da menor ocorrência de rodízio por dor e melhor controle álgico obtido após.

Outro aspecto do manejo da dor não abordado no presente trabalho e nos demais citados é a participação da equipe multiprofissional. Na unidade em estudo, participam diretamente da assistência médicos, enfermeiros, técnicos de enfermagem, psicólogos, fisioterapeutas, assistentes sociais, nutricionistas e farmacêutico, além de voluntários da capelania. Com isso, a dor pode ser abordada em todos os seus aspectos.

Os rodízios de opioide motivados por suspeiçáo de efeito adverso da medicação apresentaram pouca resposta positiva, tendo benefício em apenas dois episódios (33\%). Tal observação também foi descrita por outros autores ${ }^{11}$. Isso pode ser justificado pela suspeita de um efeito adverso de medicaçáo, quando na verdade o sintoma era decorrente da doença de base (como delirium, sonolência, náuseas etc.).
Embora a OMS ${ }^{15}$ tenha destacado a ausência de evidências para recomendaçóes de um protocolo para a prática do rodízio de opioide, um painel de especialistas sugere redução de $25 \%$ a $50 \%$ da dose equivalente de opioide ao realizar o rodízio. Nos casos de dor não controlada, recomenda-se a reduçâo entre $5 \%$ a $15 \%{ }^{3}$.

Um estudo multicêntrico observou doses equivalentes entre os opioides antes e após o rodízio, porém levou em conta a dose inicial após o rodízio, não avaliando posteriores ajustes ${ }^{11}$. Mercadante et al. ${ }^{12}$ observaram a necessidade de aumento médio na primeira semana de $23 \%$ da dose inicial do novo opioide, não ficando claro se a dose inicial no momento do rodízio foi equivalente. No presente trabalho, foi encontrada dose de destino superior. Entretanto, foi comparada com a prescrição pela rotina no dia seguinte, que inclui eventuais ajustes feitos pela equipe de plantáo ao longo do dia em que foi realizado o rodízio. Cabe ressaltar que náo houve relato de suspeita de intoxicaçáo por opioide ao longo do período estudado.

$\mathrm{O}$ aumento da dose observada no rodízio no presente estudo deve ser visto com cautela. Embora o viés na coleta possa justificar esse achado, os protocolos são unânimes em sugerir redução na dose equivalente. Os achados não são suficientes para modificar a orientação atual.

Uma avaliação da equipotência analgésica imediatamente após o rodízio deve ser realizada na unidade, para definir se o aumento observado foi decorrente de ajustes necessários e realizados nas horas subsequentes à troca do medicamento pelo médico de plantão, ou se as diretrizes não foram seguidas. Em sendo a primeira hipótese, a eficiência do trabalho da equipe clínica deverá ser destacada pela contínua avaliação do paciente para o pronto controle do sintoma. Por outro lado, se o aumento da dose equivalente do novo medicamento tiver sido prescrito de imediato, um treinamento deve ser realizado em todo o corpo clínico. $\mathrm{O}$ fato de não ter havido suspeição de intoxicação não pode ser referência para a não investigação do dado observado.

$\mathrm{Na}$ unidade de cuidados paliativos em estudo, os pacientes recebem toda medicação analgésica necessária para uso domiciliar, desde que padronizada na unidade. $\mathrm{E}$, nesse contexto, é o principal motivo de internação. O controle domiciliar da dor precisa ser incrementado e um dos aspectos básicos é o acesso a medicamentos analgésicos, em especial os opioides ${ }^{16}$. A internaçáo hospitalar, além de onerar o sistema de saúde, traz prejuízo à qualidade de vida ${ }^{17}$. Com perspectiva sobrevida curta, a luta por cada dia fora do ambiente hospitalar e com sintomas controlados deve ser buscada pela equipe de cuidados paliativos $^{18}$.

As duas principais limitaçóes deste estudo foram a coleta da dose do opioide apenas na prescrição do dia 
seguinte ao rodízio e não imediatamente, e a avaliação da eficácia do rodízio restrita a informaçóes obtidas por prontuário e náo diretamente com o paciente.

Além disso, só foram buscados desfechos em relação ao controle de dor e à suspeita de efeito adverso. Um estudo com avaliação da dose, segundo a equipotência analgésica imediatamente antes e após o rodízio, com monitorização de eventual efeito adverso ou sinais e sintomas precoces intoxicação, é sugerido na unidade.

\section{CONCLUSÃO}

Embora o controle de dor tenha sido superior ao descrito por outros trabalhos, o aumento da dose equipotente do opioide não é corroborado por protocolos. Maior vigilância e outros estudos sáo recomendados na unidade.

\section{CONTRIBUIÇÕES}

Todos os autores contribuíram na concepção e/ ou no planejamento do estudo; na obtenção, análise e interpretação dos dados; assim como na redação e revisão crítica; e aprovaram a versão final a ser publicada.

\section{DECLARAÇÃO DE CONFLITO DE INTERESSES}

Nada a declarar.

\section{FONTES DE FINANCIAMENTO}

Não há.

\section{REFERÊNCIAS}

1. Albert RH. End-of-life care: managing common symptoms. Am Fam Physician. 2017;95(6):356-61.

2. World Health Organization. National cancer control programmes: policies and managerial guidelines. 2nd ed. Geneva: WHO; 2002.

3. Fine PG, Portenoy RK. Establishing "best practices" for opioid rotation: conclusions of an expert panel. J Pain Symptom Manage. 2009;38(3):418-25. doi: https://doi. org/10.1016/j.jpainsymman.2009.06.002

4. National Comprehensive Cancer Network. Adult Cancer Pain: NCCN Guidelines. Rockledge (PA): NCCN; 2018.

5. Syrmis W, Good P, Wootton J, et al. Opioid conversion ratios used in palliative care: is there an Australian consensus? Intern Med J. 2014;44(5):483-9. doi: https:// doi.org/10.1111/imj.12401

6. Sampaio SGSM, Motta L, Caldas CP. Medicamentos e controle de dor: experiência de um centro de referência em cuidados paliativos no Brasil. Rev Bras Cancerol. 2019;65(2):e-13365. doi: https://doi. org/10.32635/2176-9745.RBC.2019v65n2.365

7. Fortunato JGS, Furtado MS, Hirabae LFA, et al. Escalas de dor no paciente crítico: uma revisão integrativa. Rev Hosp Univ Pedro Ernesto. 2013;12(3):110-7. doi: https://doi.org/10.12957/rhupe.2013.7538

8. Hennemann-Krause L. Dor no fim da vida: avaliar para tratar. Rev Hosp Univ Pedro Ernesto. 2012;11(2):26-31.

9. Jeter K, Blackwell S, Burke L, et al. Cancer symptom scale preferences: does one size fit all? BMJ Support Palliat Care. 2018;8(2):198-203. doi: https://doi.org/10.1136/ bmjspcare-2015-001018

10. Hjermstad MJ, Fayers PM, Haugen DF, et al. Studies comparing numerical rating scales, verbal rating scales, and visual analogue scales for assessment of pain intensity in adults: a systematic literature review. J Pain Symptom Manage. 2011;41(6):1073-93. doi: https:// doi.org/10.1016/j.jpainsymman.2010.08.016

11. Corli O, Roberto A, Corsi N. Opioid switching and variability in response in pain cancer patients. Support Care Cancer. 2019;27(6):2321-7. doi: https://doi. org/10.1007/s00520-018-4485-6

12. Mercadante S, Valle A, Porzio G, et al. Opioid switching in patients with advanced cancer followed at home. a retrospective analysis. J Pain Symptom Manage. 2013;45(2):298-304. doi: https://doi.org/10.1016/j. jpainsymman.2012.02.025

13. Reddy A, Yennurajalingam S, Pulivarthi K, et al. Frequency, outcome, and predictors of success within 6 weeks of an opioid rotation among outpatients with cancer receiving strong opioids. Oncologist. 2013;18(2):212-20. doi: https://doi.org/10.1634/ theoncologist.2012-0269

14. Lawlor PG, Turner KS, Hanson J, et al. Dose ratio between morphine and methadone in patients with cancer pain: a retrospective study. Cancer. 1998;82(6):1167-73. doi: https://doi.org/10.1002/ (SICI) 1097-0142(19980315)82:6<1 167::AIDCNCR23>3.0.CO;2-3

15. World Health Organization. WHO guidelines for the pharmacological and radiotherapeutic management of cancer pain in adults and adolescents. Geneve: WHO; 2019.

16. Portela FR, Moderna CM. Pacientes com câncer avançado: o acesso aos opioides e demais medicamentos para controle da dor. Rev Bras Cancerol. 2018;64(2):195201. doi: https://doi.org/10.32635/2176-9745. RBC.2018v64n2.78

17. Leira RR, Souza MFR, Costa RS, et al. Impacto econômico dos opioides no tratamento da dor oncológica em pacientes em cuidados paliativos na assistência domiciliar. Rev Bras Cancerol. 2016;62(3):271-278. doi: https://doi. org/10.32635/2176-9745.RBC.2016v62n3.344 
18. Sampaio SGSM, Motta LB, Caldas CP. Value-based medicine and palliative care: how do they converge? Expert Rev Pharmacoecon Outcomes Res. 2019:19(5):509-15. doi: https://doi.org/10.1080/14737167.2019.1651645

Recebido em 4/8/2020

Aprovado em 16/10/2020 